\title{
THE SCALE LENGTH OF OH AND CN IN COMET BENNETT (1970 II)
}

H. U. Keller and C. F Lillie

Comet Bennett (1970II) was observed with an ultraviolet spectrometer on the OAO-2 spacecraft during April 1970. The instrument consisted of a plane grating, a parabolic mirror, a slit, and a photomultiplier tube. The exit slit provided a $2^{\prime} \times 8^{\prime}$ rectangular field-of-view and a 23 A bandpass At this resolution emission features such as $\mathrm{OH} \lambda 3090$ and $\mathrm{CN}$ $\lambda 3883$ were essentially monochromatic. Since the instrument had no entrance slit, movement of the grating produced a shift in the field of view, with 1 arc min of spatial offset corresponding to a 10 A wavelength shift

The strongest cometary UV emission feature was the $(0,0)$ band of $\mathrm{OH}$ at $3090 \mathrm{~A}$ Figure 1 presents a comparison of six observed intensity profiles of $\mathrm{OH}$ with calculations based on Haser's (1957) parent-daughter-molecule model with purely radial outflow. The observed $\mathrm{OH}$ coma had an extent of $\sim 20^{\prime}$ or a diameter of $9 \times 10^{5} \mathrm{~km}$ when the comet's heliocentric and geocentric distances were about 0.8 a $u$ and 1 a.u., respectively. The scale length of the parent molecule of $\mathrm{OH}$ could not be determined, but a lower limit of $5 \times 10^{4} \mathrm{~km}$ was indicated.

The $\mathrm{OH}$ scale length, however, was, for the first time, determined to $2(+05-1.0) \times 10^{5} \mathrm{~km}$ at a cometary helio- 


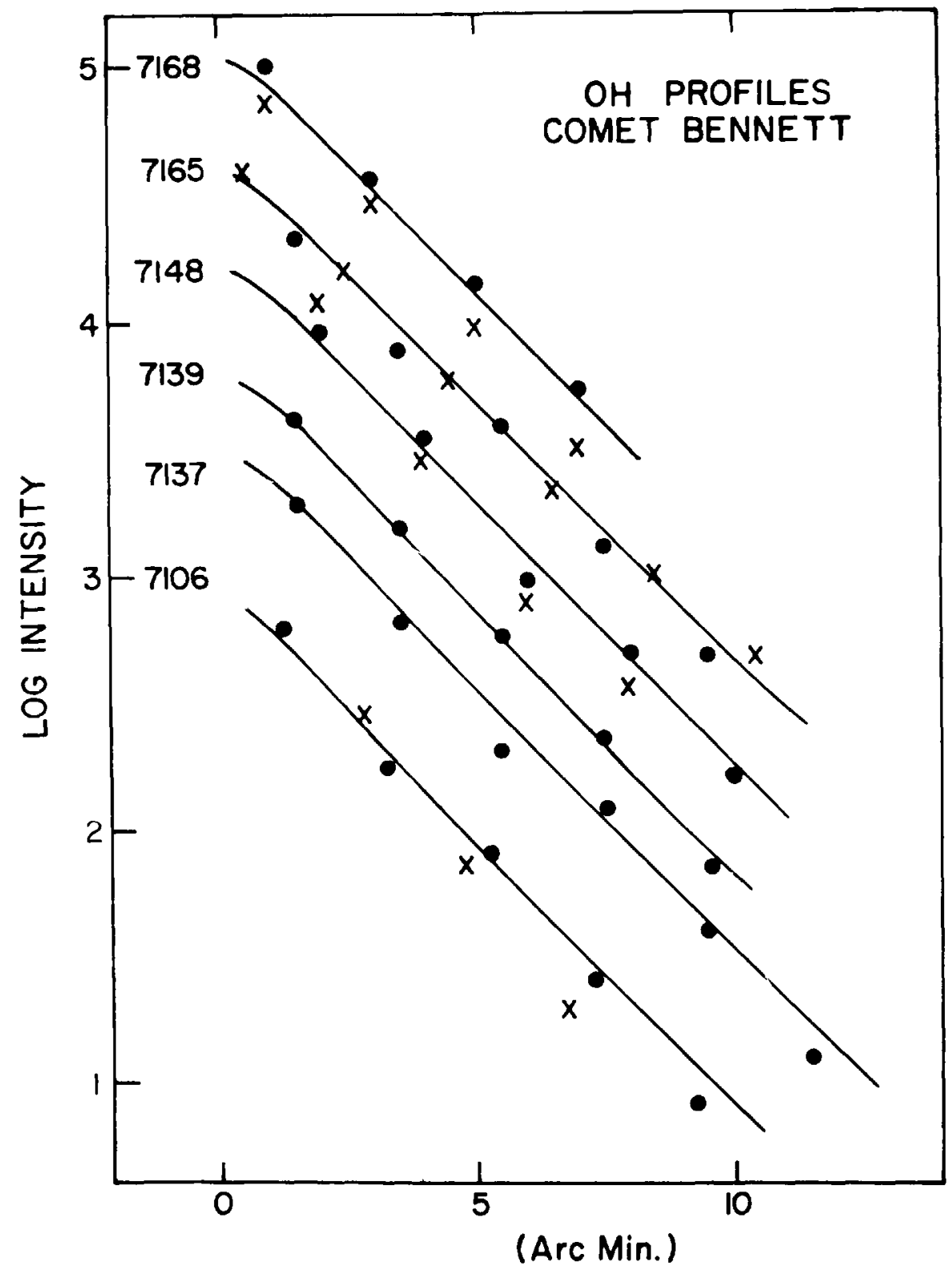

Figure 1 A comparison of model calculations with $\mathrm{OH}$ ohservations for six orbits in April 1970 The long wavelength wing is indicated by filled circles (•) while the short wavelength (sun-ward) wing is indicated with $(\mathrm{X})$ The theoretical profiles (solid line) are for scale lengths of the parent molecule of $10^{5} \mathrm{~km}$ and $2 \times 10^{5} \mathrm{~km}$ for the $\mathrm{OH}$ radical. 
centric distance of 1 a.u. This value is smaller than expected. The photodissociation cross section of $\mathrm{OH}$ is too small to yield such a short scale length (Dalgarno 1974) Since an excitation into high rotational energy levels of $\mathrm{OH}$ is not observed, one would also expect the efficiency of predissociation to be small (Smith 1970) The results presented for the $\mathrm{OH}$ scale length need further theoretical explanation.

Similar observations were made of the (0-0) $\mathrm{CN}$ band at 3883 A which is less intense than the $\mathrm{OH}$ emission. Good agreement between the observed and calculated profiles was obtained using a CN scale length of $1.4 \times 10^{5} \mathrm{~km}$ (compare Delsemme and Moreau 1973).

The results are presented in more detail by Keller and Lillie (1974) 


\section{REFERENCES}

Dalgarno, A. 1974, private communication.

Delsemme, A. H., Moreau, J. L. 1973, Astrophys Letters 14, 181 .

Haser, L. 1957, Bull. Acad. Roy Sci. Belgique 43, 740.

Keller, H. U., Lillie, C. F. 1974, Astron. and Astrophys 34, 187.

Smith, W. H. 1970, J Chem. Phys 53, 792. 